\title{
Asymmetrical Spotting and Direction of Circling in the Varitint-Waddler Mouse
}

\author{
A. R. CoOLs
}

$\mathbf{I}^{\mathrm{N}}$ $\mathrm{N}$ the mouse (Mus musculus) many genes are known in which the aberrant allele leads to the waltzing-shaker syndrome of circling, headtossing, and deafness ${ }^{10}$. A semidominant type of these neurological mutants is the varitint-waddler $(V a)^{3,10}$. Apart from these disorders the $V a$ syndrome involves variegation of the fur, postnatal degeneration of the inner ear, and abnormal behavioral components $^{6, i}$. Although the deafness is fully explained by the cochlear defect, the rotational behavior and other behavioral abnormalities are not clearly understood. It has been suggested that the hyperactivity of the $V a$ mice, together with their life in a confining mouse cage, may lead to circling ${ }^{\top}$. However, no experiments have tested this hypothesis.

In order to examine the relationship between circling and other symptoms of the $V a$ syndrome, a study was made of 1 ) the development of 15 reflexes during the critical transition period of neurological maturation, 2) the open-field behavior of adults, and 3 ) the influence of available space during growth on circling and locomotor activity.

During preliminary experiments a remarkable relation was observed between the dominant direction of circling and the location of non-fading pigment areas on the fur. Since neural crest cells, from which the melanocytes are derived, are the primary source of several neural structures, it was tempting to postulate that the laterality in circling and the asymmetry in the fur pattern are both expressions of the same basic neurological disturbance. A study, therefore, was included of this relationship.

Our results suggest that a disorder of the central nervous system is involved in the waltzer-shaker syndrome of the varitint-waddler. This may involve a disturbance of dopamine synthesis in the striatal region.

\section{Materials and Methods}

Heterozygous $\mathrm{Va}$ mice were used routinely in this study, since homozygous $V a$ mice usually die before

This work was accomplished during the author's affiliation with the Laboratory of Genetics, University of Nijmegen, The Netherlands. The author's present address is Pharmacological Laboratory, Faculty of Medicine, University of Nijmegen. one month of age. The $\mathrm{Va}$ strain was obtained from Dr. D.S. Falconer, Institute of Animal Genetics, Edinburgh. Although the strain with the $V a$ gene was crossed with different mutant strains in our laboratory, the animals had been outbred only by sib mating with animals from inbred strain C57BL/ $6 \mathrm{~J}$ during the last five years. Nonmutant littermates were used as controls.

Wild-type and mutant mice were kept with their littermates in large perspex cages $(32 \times 21 \times 16$ $\mathrm{cm}$ ) until the 30th postnatal day. In order to ensure some uniformity in nutrition and growth rate, the litter size was reduced to 7 or 8 animals. On the 30th day, 18 wild-type mice and 18 mutant mice were isolated in small perspex cages $(13 \times 21 \times 16$ $\mathrm{cm})$. Thirteen other mutant animals were isolated in large cages $(32 \times 21 \times 16)$. Temperature, day/ night periodicity, and light intensity were standardized; food and water were available ad libitum, except during the tests.

\section{Reflex patterns}

Ten male wild-type and ten $V a$ mice were subjected to the following reflex tests during the 7th, 8th, 14th, and 15th postnatal day: righting reflex (a), crossed extensor reflex (b), forelimb placing (c), hindlimb placing (d), postural flexion (e), postural extension (f), pivoting (g), straight line walking (h), grasp reflex (i), rooting reflex (j), vibrissae placing ( $k$ ), visual placing (1), negative geotaxis (m), bar holding (n), and cliff drop aversion (o). For a description of these reflexes reference is made to Fox. The rating method was modified so that all reflexes were recorded in a standard fashion by means of numerical scoring from $0,1,2$, 4,6 , to 8 , where $0=$ no response and $8=$ maximum response. Each figure corresponded to an operationally defined response in order to acquire an objective measure. For example, in the crossed extensor reflex, $0=$ no response; $1=$ one hindlimb flexed; $2=$ two hindlimbs flexed; $4=$ one hindlimb flexed and extended; $6=$ one hindlimb flexed and extended, the other flexed; and $8=$ two hindiimbs flexed and extended. Similar gradations were used for all responses. A more detailed description is given elsewhere ${ }^{2}$. Every mouse was tested once for each given test. For the mice of each mutant and non- 
mutant group, all scores were added up in order to obtain a total score at each age.

\section{Open-field behavior}

When afflicted mice were 25 days old, a qualitative study of $V a$ behavior was initiated. Ten male $\mathrm{Va}$ mice that had been reared in large cages were placed singly into an observation cage and watched continuously for 15 minutes once a week over a period of 12 weeks. For a description of the observation cage, reference is made to Abeelen ${ }^{1}$. Attention was given to the presence of abnormal postures and movements during each period using a checklist of normal mouse behavior ${ }^{\mathrm{1}}$.

\section{Influence of available space on circling and loco- motor activity}

Ten male $V a$ mice reared in small cages and ten male $V a$ animals reared in large cages were tested on five successive days with respect to their circling and locomotor activity. Since $V a$ animals have the tendency to turn around obstacles, the circling activity was measured by means of a specially constructed drum. A small closed cylinder $(r=1.5$ $\mathrm{cm})$ was fixed in the center of a large one $(r=9.5$ $\mathrm{cm}$ ). The floor area in between was interrupted by two small parts $(8 \times 4 \mathrm{~cm})$ along the diameter. These portions, which were depressed by the weight of a mouse passing over them, made an electrical contact. The two switches had to be closed sequentially in order to register on a counter. Visual observation was applied to distinguish between right and left circling. The wild-type mice made an average score of 30 without any circling in a period of 15 minutes; the score of the $\mathrm{Va}$ mice, therefore, was corrected for this value.

Locomotor activity was measured in a normal T-maze during 30 minutes; the number of entrances was registered automatically. In a preliminary study it was found that both the circling and the locomotor activity of the $V a$ mice had two maxima during the day: one at 10:00-11:00 a.m., and one at 3:004:00 p.m. On the same day, when the circling activity was measured during 15 minutes at 10:00 a.m., the locomotor activity was measured during 30 minutes at 3:00 p.m.

\section{Spotting pattern and circling preference}

In preliminary experiments it was found that animals without spotting (SP) did not show any circling activity, while animals with SP showed the following relation between the distribution of the spots and the direction of circling: 1) SP in the dorsal area in front of the interaural line at one side of the midsagittal plane corresponded with circling contralateral to that area; 2) SP in the dorsal area behind the interaural line at one side of the midsagittal plane corresponded with circling homolateral to that area; 3) SP in the ventral area at one side of the midsagittal plane corresponded with circling contralateral to that area; 4) equal distribution of SP at both sides corresponded with circling without any preference; and 5) in case of unequal SP in two areas, the area with the largest amount of SP determined the predominant direction of circling.

In order to test the validity of these findings, the fur patterns of $31 \mathrm{Va}$ mice, ranging in age from 60 to 120 days, were coded using the six mentioned areas as independent elements. Each type of distribution pattern represented a theoretical value of circling direction; each spotted area was coded by $\mathbf{R}$ (turning to the right) or $\mathrm{L}$ (turning to the left). In case of equal distribution of SP between two areas, the symbol $M$ (no circling preference) was used; in case of unequal distribution of SP between two areas, the symbols RRL (turning, predominantly to the right) or RLL (turning, predominantly to the left) were used. Absence of SP was coded by the symbol $O$ (no circling activity).

The expected values were compared with experimental scores, measured in the drum by counting the clockwise and counterclockwise turnings during 15 minutes at 10:00 a.m. for five successive days. In view of the dependency of two successive circling movements, the experimental scores were evaluated by means of the quotient $\mathrm{R}-\mathrm{L} / \mathrm{R}+\mathrm{L}=(\mathrm{Y})$ :

$$
\begin{array}{r}
-0.1<\mathrm{Y}<+0.1: \text { no turning preference }(\mathrm{M}), \\
\mathrm{Y} \geq+0.1: \text { turning to the right }(\mathrm{R}), \\
\mathrm{Y}<-0.1: \text { turning to the left }(\mathrm{L}), \\
\mathrm{Y}=0 \text { : no circling }(\mathrm{O}) \text {. }
\end{array}
$$

\section{Results}

\section{Reflexes}

Differences in the responses of wild-type and $\mathrm{Va}$ mice of the same age were evaluated nonparametrically by means of the Mann-Whitney test ${ }^{18}$. In general, our data on reflex development did not deviate too much from those reported for the Nijmegen waltzers $(n v)^{2}$.

No significant differences in the righting reflex, crossed extensor reflex, forelimb placing, posture flexion and extension, and visual placing were observed between wild-type and $V a$ mice. Judging from the significant differences $(P<0.05)$ on day 8 , hindlimb placing and negative geotaxis seemed to develop more slowly in $V a$ mice than in control animals, although both genotypes approached their maximum strength on day 15 . The $V a$ score in the straight line walking test was significantly lower, since the $V a$ animals started to pivot before they had reached the endpoint of the fixed distance. The development of the above-mentioned reflexes was similar to that reported for the development of the Nijmegen waltzer ${ }^{2}$. However, there were a few deviations, notably a difference in vibrissae placing, rooting reflex, grasp reflex, and cliff drop aversion. In the vibrissae placing test the $V a$ mice showed no response in contrast to the $n v$ mice, which responded normally. The rooting response was significantly disturbed in the $V a$ mice as they showed no forward 
crawling. In the $n v$ mice, however, this response was normally developed. In the grasp reflex test, $V a$ mice did not perform any oriented grasp movement on days 8,14 , and 15 , while this response was quite well developed in $n v$ mice on day 8 . Finally, both in the bar holding and cliff drop aversion tests on day $15, \mathrm{Va}$ mice caught hold of the bar instead of walking, or held on to the edge of the table without moving, instead of turning. These tendencies were also reported for the $n v$ mice.

\section{Open-field behavior}

During all movements in the beginning of the exploring phase, the $V a$ mouse remained close to the sides of the box, with its head turned away from the wall, eyes looking upwards and offhindlimb extended. When moving through the box, the animal showed two different gaits: it walked with the hindlimbs lifted well off the ground or it fidgeted in the slinking gait. During the exploring phase retropulsion was sometimes seen. After about five minutes an increasing restlessness was shown, and the $V a$ mouse would make rapid rushes without avoiding obstacles such as the wall of the box. These rapid running movements were interrupted by short periods during which the animal remained rigid and alert, often adopting some characteristic postures: 1) it reared on its hindquarters with the forepaws raised and head swaying, or 2) it remained motionless in the splay-leg or in the hunched posture with tailraising and headtossing. When headtossing was observed, vertical movements predominated. With respect to the waltzing syndrome, only the animals with SP showed spinning or running in circles and figureeights. At the end of 10 minutes, the animals were creeping, skipping, or leaping within the box, without any orientation. Occasionally there were clonic convulsive movements during this period.

All these postures and movements were present

Table I. Median scores of circling and locomotor activity of $\mathrm{Va} /+$ mice reared in small and large cages

\begin{tabular}{ccccc}
\hline \multicolumn{2}{c}{ Locomotor activity* } & & \multicolumn{2}{c}{ Circling activity** } \\
\cline { 1 - 1 } \cline { 5 - 5 } Small cage & Large cage & & Small cage & Large cage \\
\hline 113 & 372 & & 16 & 370 \\
498 & 119 & & 664 & 71 \\
711 & 642 & & 304 & 504 \\
406 & 381 & & 253 & 103 \\
315 & 858 & & 359 & 137 \\
814 & 467 & & 561 & 63 \\
370 & 938 & & 22 & 589 \\
742 & 648 & & 410 & 551 \\
302 & 527 & & 463 & 483 \\
978 & 456 & & 568 & 409 \\
& & &
\end{tabular}

* Small cage-reared vs. large cage-reared: not significant at $P<0.05$ (Wilcoxon test)

** Small cage reared vs large cage reared : not significant at $P<0.05$ (Wilcoxon test) during each observation period, and were highly characteristic of $V a$ mice. Apart from the aberrant behavior, $\mathrm{Va}$ mice showed quite normal activity, i.e., reconnoitering, cleaning, sniffing, walking, and springing occurred in the second period of five minutes. Since only the presence and not the frequency of the components was recorded, nothing can be said of the quantitative aspects of these components.

\section{Influence of available space on circling and locomotion}

Differences in the circling and locomotor activity between $V a$ mice reared in small cages and those reared in large cages were evaluated by means of the Wilcoxon test or the Fisher exact test ${ }^{18}$. All the median scores are summarized in Table I. No significant differences between the two groups were observed in terms of circling behavior and locomotor activity.

\section{Spotting pattern and circling preference}

The total code of expected circling behavior for each animal, depending on the location and number of spotted areas, is given in the left column of Table II, while the experimental scores of five experiments, calculated with the quotient $\mathrm{R}-\mathrm{L} / \mathrm{R}+\mathrm{L}=(\mathrm{Y})$, are summarized in the right column of Table II. As predicted, unspotted animals showed no circling activity. All 10 animals with one spotted area gave the predicted scores in at least four tests. Of the eight animals that had two spotted areas, six turned to the expected side, one (no. 25) scored successfully in two tests and nearly successfully $(0<\mathrm{Y}<$ - 0.1) in two others, and one animal (no. 19) turned to the nonexpected side. All five animals with three spotted areas turned to the expected dominant side in four tests. The animal with four spotted. areas did not turn at all, although circling without preference was predicted.

\section{Discussion}

Three kinds of reflexes are present in the varitintwaddler: 1) normally developed reflexes (nos. a, b, $\mathrm{c}, \mathrm{e}, \mathrm{f}$, and 1$)$; 2) reflexes in which development is retarded (nos. $d$ and $\mathrm{m}$ ); and 3 ) reflexes in which development is disturbed (nos. $\mathrm{g}, \mathrm{h}, \mathrm{i}, \mathrm{j}, \mathrm{k}, \mathrm{n}$, and o) .

Since the labyrinth itself is strongly disturbed at the age of 8 days $^{\tau}$, the normal development of the righting reflex-a labyrinth reflex-may express the existence of a secondary adaptation mechanism. The nearly normal development of hindlimb placing and negative geotaxis on day 15 , following the original retardation, strengthens this assumption.

The most important findings, however, seem to be the abnormal development of the grasp and rooting reflexes: the labyrinth is not involved in these responses. These anomalies, combined with the defects in vibrissae placing, pivoting, and straight line walking, seem to indicate that a second 
disorder, central in origin, exists in the varitintwaddler.

With respect to the behavior of adult $\mathrm{Va}$ mice, three conclusions can be made. First, there is good agreement with the description of $\mathrm{Va}$ behavior given by Cloudman and Bunker. ${ }^{3}$. Second, the presence of behavioral patterns such as walking and reconnoitering on the one hand, and the temporary appearance of such behavior patterns as headtossing, circling, and fidgeting on the other hand indicate that the central mechanism of integration and coordination with respect to posture and locomotion is involved in these temporary disturbances. Third, the observed behavior of $\mathrm{Va}$ mice shows an impressive resemblance to the behavior of normal mice injected with high doses of amphetamine ${ }^{11}$, e.g., "the animals are extremely restless, make frequent rapid rushes often accompanied by squealing, and sometimes sit up on their hind quarters with inquisitive rotational movements of the head and neck. Occasionally there are clonic convulsive movements." The importance of the amphetamine mimicry will be mentioned below.

From the absence of any significant influence of the available space on rotational behavior and hyperactivity, it can be concluded that the circling activity is certainly not the product of hyperactivity and growing up in the confined space of a mouse cage as suggested in the literature ${ }^{\top}$. In fact, both activities seem to be independent of this external factor.

Otherwise, the relative frequency of animals having a specific circling preference (71 percent) and the relation between the laterality in circling and the asymmetry in nonfading spots point rather to the involvement of a more fundamental disorder. Although a definite explanation for the laterality or for the asymmetry of the nonfading pigment spots is not available ${ }^{16}$, the finding that 93 percent of the experimental circling values corresponded with the expected circling values suggests that both expressions may be causally related to one basic mechanism.

The pigment spots of the skin are filled up by melanocytes ${ }^{17}$, in which melanin is formed through the oxidation of 3,4-dihydroxyphenylalanine (DOPA). The abnormal piebald spotting pattern with nonfading spots on a fading background may be most simply explained by a disorder of the melanin-synthesizing mechanism, although an alternative explanation might be the existence of a high somatic mutation rate of the gene ${ }^{16,17}$.

On the other hand, anomalies such as abnormally developed reflexes, hyperactivity, temporary presence of abnormal postures and movements, and circling and disoriented behavior point to a disorder central in origin. In this context, it is noteworthy that dihydroxyphenylalanine is not only the precursor of melanin, but also of dopamine. The highest concentration of dopamine within the brain is found in the synapses of the corpus striatum ${ }^{12}$; it fulfills an essential function with respect to the integration and coordination of motor activity ${ }^{4,12}$. Furthermore, circling, hyperactivity, and movements of the head and the limbs can be elicited by electrical or chemical stimulation of the corpus striatum ${ }^{4,9,12,13,19}$. Recently, Potegal has shown that the corpus striatum is involved in the mechanism of orientation ${ }^{15}$; this mechanism is strongly disturbed in the varitintwaddler. Indeed, Deol has already mentioned that the shaking movements of the head may be analogous to Huntington's chorea in man, in which similar head movements are held to be due to the involvement of the striatal system ${ }^{6}$. Furthermore, the waltzingshaker syndrome of shaker-1 mice is also associated with a disorder of the striatal system ${ }^{20}$.

Summarizing all these data, we find neurological

Table II. Expected and observed values of circling preference in $31 \mathrm{Va} /+$ mice*

\begin{tabular}{|c|c|c|}
\hline Animals & Exp. Pref. (X) & $\begin{array}{c}\text { Obs. pref. (Y) } \\
\text { (outcome of } 5 \text { exp.) }\end{array}$ \\
\hline \multicolumn{3}{|c|}{ No spotting } \\
\hline 1 & 0 & $0-0-0-0-0$ \\
\hline 2 & 0 & $0-0-0-0-0$ \\
\hline 3 & 0 & $\mathrm{O}-\mathrm{O}-\mathrm{O}-\mathrm{O}-\mathrm{O}$ \\
\hline 4 & 0 & $\mathrm{O}-\mathrm{O}-\mathrm{O}-\mathrm{O}-\mathrm{O}$ \\
\hline 5 & 0 & $0-0-0-0-0$ \\
\hline 6 & 0 & $0-0-0-0-0$ \\
\hline 7 & 0 & $\mathrm{O}-\mathrm{O}-\mathrm{O}-\mathrm{O}-\mathrm{O}$ \\
\hline \multicolumn{3}{|c|}{ One spotted area } \\
\hline 8 & $\mathbf{R}$ & $\mathbf{R}-\mathbf{R}-\mathbf{R}-\mathbf{R}-\mathbf{M}$ \\
\hline 9 & L & $\mathbf{L}-\mathbf{L}-\mathbf{L}-\mathbf{L}-\mathbf{L}$ \\
\hline 10 & $\mathbf{R}$ & $\mathbf{R}-\mathbf{R}-\mathbf{R}-\mathbf{R}-\mathbf{R}$ \\
\hline 11 & $\mathbf{R}$ & $\mathbf{R}-\mathbf{R}-\mathbf{R}-\mathbf{R}-\mathbf{R}$ \\
\hline 12 & $\mathrm{~L}$ & $\mathrm{~L}-\mathrm{L}-\mathrm{L}-\mathrm{L}-\mathrm{L}$ \\
\hline 13 & $\mathbf{R}$ & $\mathbf{R}-\mathbf{R}-\mathbf{R}-\mathbf{R}-\mathbf{R}$ \\
\hline 14 & $\mathbf{R}$ & $\mathbf{R}-\mathbf{R}-\mathbf{R}-\mathbf{R}-\mathbf{R}$ \\
\hline 15 & $\mathbf{R}$ & $\mathbf{R}-\mathbf{R}-\mathbf{R}-\mathbf{R}-\mathbf{R}$ \\
\hline 16 & $\mathrm{~L}$ & $\mathbf{L}-\mathbf{L}-\mathbf{L}-\mathbf{L}-\mathbf{L}$ \\
\hline 17 & $\mathbf{R}$ & $\mathbf{R}-\mathbf{R}-\mathbf{R}-\mathbf{R}-\mathbf{R}$ \\
\hline \multicolumn{3}{|c|}{ Two spotted areas } \\
\hline 18 & RLL & $\mathrm{L}-\mathrm{L}-\mathrm{L}-\mathbf{L}-\mathbf{L}$ \\
\hline $19+$ & RLL & $\overline{\mathbf{R}}-\overline{\mathbf{R}}-\mathbf{R}-\mathbf{R}-\mathbf{R}$ \\
\hline 20 & RRL & $\mathbf{R}-\mathbf{R}-\mathbf{R}-\mathbf{R}-\mathbf{L}$ \\
\hline 21 & $\mathbf{R R}$ & $\mathbf{R}-\mathbf{R}-\mathbf{R}-\mathbf{R}-\mathbf{M}$ \\
\hline 22 & $\mathrm{RLL}$ & $\mathbf{R}-\mathbf{R}-\mathbf{L}-\mathbf{L}-\mathbf{L}$ \\
\hline 23 & M & $\mathrm{M}-\mathrm{M}-\mathrm{M}-\mathrm{M}-\mathrm{O}$ \\
\hline 24 & RLL & $\mathrm{R}-\mathrm{L}-\mathrm{L}-\mathrm{L}-\mathrm{L}$ \\
\hline $25 \dagger$ & RLL & $\mathbf{R}-\mathbf{L}-\mathbf{L}-\mathbf{M}-\mathbf{M}$ \\
\hline \multicolumn{3}{|c|}{ Three spotted areas } \\
\hline 26 & R.R.R. & $\mathbf{R}-\mathbf{R}-\mathbf{R}-\mathbf{R}-\mathbf{O}$ \\
\hline 27 & R.RRL & $\mathbf{R}-\mathbf{R}-\mathbf{R}-\mathbf{R}-\mathbf{L}$ \\
\hline 28 & R.RRL & $\mathbf{R}-\mathbf{R}-\mathbf{R}-\mathbf{R}-\mathbf{R}$ \\
\hline 29 & RLL.L & $\mathrm{R}-\mathrm{L}-\mathrm{L}-\mathrm{L}-\mathrm{L}$ \\
\hline 30 & R.RRL & $\mathrm{R}-\mathrm{R}-\mathbf{R}-\mathrm{R}-\mathrm{L}$ \\
\hline \multicolumn{3}{|c|}{ Four spotted areas } \\
\hline $31 \dagger$ & M.M & $0-0-0-0-0$ \\
\hline
\end{tabular}

* For each animal the total code of expected circling behavior, depending on the location and the number of spotted areas, is given in the left column, while the scores of circling activity, measured in five tests, and calculated with the quotient $\mathrm{R}-\mathrm{L} / \mathrm{R}+\mathrm{L}$, are given in the right column. $\mathrm{R}=$ turning to the right; $L=$ turning to the left; $M=$ no turning preference; $O=$ absence of circling; RRL = turning, predominantly to the right, and $R L L=$ turning, predominantly to the left

$\dagger$ No agreement between the expected and observed values of the circling preference 
symptoms characteristic of a striatal involvement, and a localized pigmentary defect whose position is correlated with the direction of the abnormal circling movements. Furthermore, dopamine, known to be important in the corpus striatum, is chemically related to melanin, which is abnormally synthesized in the varitint-waddler. It is reasonable to suggest that the pigmentary disorder and the dyskinesia are causally related to a single underlying dopaminesynthesizing mechanism that is under the control of the $V a$ locus. The previously mentioned behavioral similarity of $V a$ mice to amphetamine-treated normal mice strengthens this assumption, since the effect of amphetamine is primarily upon dopamine in the corpus striatum ${ }^{14}$. A psychopharmacological study, presenting evidence in support of this hypothesis, has been published elsewhere ${ }^{5}$.

\section{Summary}

The relationship between the circling trait and other symptoms such as deafness, hyperactivity, and headtossing of the waltzing-shaker syndrome in the varitintwaddler mouse was investigated. An ontogenetic test of 15 reflexes showed normally and abnormally developed reflexes; some of the last were independent of the labyrinth disorder. An open-field test revealed temporary disturbances of normal postures and locomotor patterns in adults. A third test indicated that the development of both circling and locomotor activity was independent of the dimension of the available space during growth. Analysis of the direction of circling and the variegation of the fur showed an impressive relation between the laterality in circling and the asymmetry of the nonfading spots on the fur. The involvement of a striatal disorder, based on a disturbance of dopamine synthesis, is discussed.

\section{Literature Cited}

1. Abeelen, J. H. F. van, Behavioral profiles of neurological mutant mice. Genetica 37:149-159. 1966.

2. - and J. TH. R. KALKhoven. Behavioral ontogeny of the Nijmegen waltzer, a neurological mutant in the mouse. Animal Behav. 18:711-718. 1970.
3. Cloudman, A. M. and L. F. Bunker, JR. The varitintwaddler mouse: a dominant mutation in Mus musculus. $J$. Hered. 36:259-263. 1945.

4. Cools, A. R. and J. M. van Rossum. Caudal dopamine and stereotype behaviour of cats. Arch. int. Pharmacodyn. 187:163-173. 1970.

5. Cools, A. R. Neurochemical correlates of the waltzingshaker syndrome in the varitint-waddler mouse. Psychopharm. 24:384-396. 1972.

6. Deol, M. S. The anomalies of the labyrinth of the mutants varitint-waddler, shaker-2, and yerker in the mouse. J. Genet. 52: 562-588. 1954.

7. - The probable mode of gene action in the circling mutants of the mouse. Genet. Res. 7:363-371. 1966.

8. Fox, W. M. Reflex-ontogeny and behavioral development of the mouse. Animal Behav. 13:2-3. 1965.

9. Grossman, S. P. A Textbook of Physiological Psychology. John Wiley \& Sons, Inc., New York. p. 241-256. 1967.

10. GrünEberg, $H$. The Genetics of the Mouse. Martinus Nijhoff, The Hague. p. 193-200. 1952.

11. GunN, J. A. and M. R. GuRd. The action of some amines related to adrenaline: cyclohexylalkylamines. $J$. Physiol. 97: 453-470. 1939.

12. HornYKIEwICZ, O. J. Dopamine (3,-hydroxytyramine) and brain disfunction. Pharmacol. Rev. 18:925-964. 1966.

13. GiJbels, J. Het gedrag van de kat na letsels van de nc caudatus. Verh. Kon. Vlaamse Acad. Geneesk. Belgium 77:287-349. 1965.

14. McKenzie, G. M. and J. C. Szerb. The effect of dihydroxyphenylalanine, pheniprazine and dextroamphetamine on the in vivo release of dopamine from the caudate nucleus. J. Pharmacol. Exp. Ther. 162:302. 1968.

15. Potegal, M. Role of the caudate nucleus in spatial orientation of rats. J. Comp. Physiol. Psychol. 69:756. 1969.

16. Schaible, R. H. Clonal distribution of melanocytes in piebald-spotted and variegated mice. J. Exp. Zool. 172:181200. 1969.

17. SeArle, A. G. Comparative Genetics of Coat Colour in Mammals, Logos/Academic, London/New York. p. 4554. 1968.

18. Siegel, S. Non-Parametric Statistics for the Behavioral Sciences. McGraw-Hill, New York, p. 68. 1956.

19. White, R. P. and H. F. Himwich. Ablation study on the mechanism of forced circling in the rabbit. $A m$.J. Physiol. 179:684. 1954.

20. ZimMERMAN, K. Erbliche Gehimerkrankungen der Hausmaus. Erbarzt (Beil, z. Dtsch. Aerztebl. 1935, 2). p. 119-120. 1935. 